\title{
SIMULAÇÃO NUMÉRICA NÃO-LINEAR DO COMPORTAMENTO DE PLACAS DE AÇO PERFURADAS SOB COMPRESSÃO UNIAXIAL EM ESTRUTURAS NAVAIS E OFFSHORE
}

\author{
Lillian Gonçalves Baptista ${ }^{1}$ \\ Mauro de Vasconcellos Real ${ }^{2}$ \\ Liércio André Isoldi ${ }^{3}$
}

\begin{abstract}
Resumo: Placas são elementos estruturais de superfície plana amplamente empregados em engenharia, cuja espessura é pequena quando comparada com as demais dimensões. Estas estruturas laminares podem ser delimitadas por linhas retas, denominadas enrijecedores, formando parte integrante de estruturas de navios, plataformas de petróleo offshore, comportas e docas flutuantes. É muito comum encontrar perfurações nessas placas para inspeção e manutenção, e o tamanho desses furos tem significativa importância no desempenho da estrutura. Uma análise não-linear da flambagem com base no método dos elementos finitos, considerando-se as imperfeições geométricas iniciais e as nãolinearidades do material, foi realizada para um grupo de 150 placas onde a largura é mantida constante e o comprimento é variado, assim como a espessura e o diâmetro do furo circular centralizado. A modelagem computacional foi realizada no software ANSYS, onde foi possível obter a carga de ruptura última para cada modelo e analisar a influência do tamanho do furo, da esbeltez e do comprimento variável na capacidade de carga das placas. Ficou evidente que a força máxima é significativamente reduzida pelo furo. Nota-se também que quanto mais espessa a placa, maior sua resistência, que a capacidade de carga é raramente afetada com a variação de seu comprimento.
\end{abstract}

Palavras-chave: flambagem deplacas, método dos elementos finitos, simulação numérica

Abstract: Plates are flat surface structural elements widely used in engineering, whose thickness is small compared to other dimensions. These laminar structures can be bounded by straight lines, called stiffeners, forming part of structures of ships, offshore oil platforms gates and floating docks. It is very common to find holes in these plates for inspection and maintenance, and the size of these holes hasgreat importance on the structure performance. A non-linear buckling analysis, based on the finite element method, considering the initial geometric imperfections and material non-linearity was performed for a group of 150 plates where the widthwas kept constant and itslength was varied, as well asthe thickness and the diameter of the central circular hole. The computer modeling was performed using ANSYS software.It was possible to get the ultimate strength for each plate model and analyze the influence of the holesize, slenderness and variable length on the load carrying capacity of the plates. It was evident that the maximum strength is significantly reduced by the hole. It was also noted that the thicker the plate, the greater its resistance, but the load capacity is rarely affected by the variation of the plate length.

Keywords: buckling of plates, finite element method, numerical simulation

\footnotetext{
${ }^{1}$ Universidade Federal do Rio Grande - FURG. E-mail: lbaptista@ furg.br

2 PPGEO, Programa de Pós-Graduação em Engenharia Oceânica, Universidade Federal do Rio Grande FURG. E-mail : mauroreal@ furg.br

3 PPGEO, Programa de Pós-Graduação em Engenharia Oceânica, Universidade Federal do Rio Grande FURG. E-mail: liercioisoldi@furg.br
} 


\section{INTRODUÇÃO}

\subsection{Considerações iniciais}

Placas finas são elementos estruturais amplamente empregados em engenharia. Este fato é explicado por diversos aspectos favoráveis ligados a estes elementos, dentre os quais se destacam a elevada relação resistência-peso e a existência de eficientes métodos analíticos descrevendo seu comportamento mecânico. As placas são caracterizadas por possuírem uma dimensão (espessura) muito menor que as outras e por desempenharem sua função estrutural sob solicitações de flexão e/ou solicitações em seu próprio plano.

Segundo Kumar (2007), as placas finas limitadas por enrijecedores são parte integrante das estruturas de navios, plataformas de petróleo offshore, comportas e docas flutuantes (Figura 1.1). Aberturas são feitas nessas placas para acesso, manutenção ou redução do peso total. A realização dessas aberturas influencia na resistência máxima desses elementos, uma vez que ocorre uma redistribuição das tensões, ocasionando uma mudança no comportamento mecânico da estrutura.

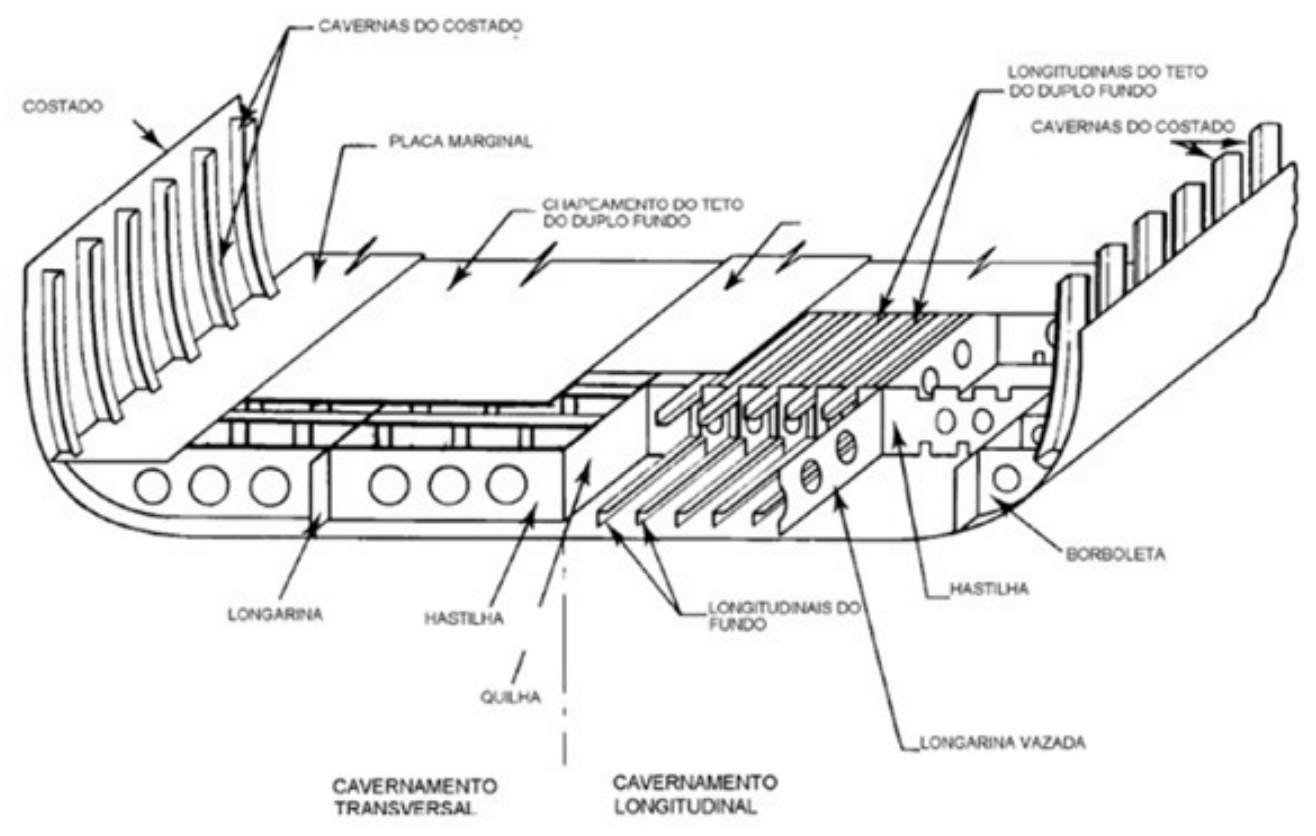

Figura 1.1 - Ilustração da estrutura terciária do duplo-fundo de um navio.

Até 1930 acreditava-se que o início da flambagem de placa implicava em seu colapso, similarmente ao que se observava no comportamento de barras comprimidas (Roorda,1980) e (Trahair e Bradford, 1988). Entretanto, com o avanço da indústria aeronáutica, diversos pesquisadores voltaram seus estudos para a instabilidade de estruturas esbeltas e concluíram que a carga de colapso de uma placa plana era realmente maior que sua carga de flambagem, mostrando que, na verdade, as placas possuem grande resistência pós-flambagem (Schuman e Back, 1959). Essa capacidade de carga pós-flambagem permite um carregamento adicional após a curvatura local e a carga de ruptura não é restrita à ocorrência da deformação elástica. $\mathrm{O}$ conhecimento desta força adicional é de grande importância prática no projeto de navios e estruturas aeroespaciais, uma vez que, considerando o comportamento pósflambagem de placas, consideráveis economias de peso podem ser obtidas.

\subsection{Revisão bibliográfica}

A investigação da flambagem de placas teve origem na observação do comportamento de placas planas de navios. Foi Bryan (1891) quem apresentou a primeira solução da equação diferencial de placa de Saint-Venant 
(1883), com a análise da tensão crítica elástica de uma placa retangular apoiada nas quatro bordas e submetida a uma tensão de compressão uniforme longitudinal.

Por volta de 1910, ainda para análise elástica, Timoshenko (1910) apresentou soluções para vários outros casos de condições de contorno. Em seguida, Bleich (1924) estendeu essa análise para o regime inelástico.

Desde então, o fenômeno da estabilidade de placas tem sido exaustivamente estudado e sintetizado por diversos autores. Narayanan eChow (1984) desenvolveram gráficos com base na capacidade final de placas perfuradas submetidas a compressão uniaxial com aberturas quadradas e circulares. Roberts e Azizian(1984) geraram as curvas de interação para a força final de placas quadradas com furos quadrados $\mathrm{e}$ circulares centrais submetidas à compressão uniaxial, compressão biaxial e cisalhamento puro. Yettrame Brown (1985) estudaram o comportamento de estabilidade de placas planas quadradas com perfurações quadradas centralizadas. Shanmugam (1997) analisou os efeitos de aberturas em elementos de placa enrijecidos submetidas à compressão uniaxial, compressão biaxial e cisalhamento puro. Shanmugam, Thevedran eTan (1999) utilizaram o Método dos Elementos Finitos e analisaram a flambagem não-linear de placas quadradas com furos centrados, quadrados e circulares. Paik, Thayamballi e Kim (2001) apresentaram formulações para a resistência última de chapeamento de navios sob a combinação de tensões de compressão biaxial, cisalhamento de borda, e cargas de pressão lateral. Uma análise elastoplástica é feita aplicando-se um deslocamento na placa e monitorando a tensão gerada nela. El-Sawy, Nazmye Martini (2004) utilizaram o método de elementos finitos para determinar a tensão de flambagem elastoplástica de placas quadradas e retangulares simplesmente apoiadas com furo circular carregadas uniaxialmente.
Apesar de o comportamento elástico de placas finas perfuradas ter recebido a atenção de vários pesquisadores nas últimas décadas, muito pouco foi reportado sobre a análise de flambagem não-linear dessas estruturas.

\subsection{Proposta de estudo}

O objetivo deste estudo numérico com base no método dos elementos finitos é realizar a modelagem computacional no software ANSYS de um grupo de 150 placas finas usualmente empregadas na construção de plataformas de petróleo offshore, de material isotrópico e homogêneo, contendo ou não perfurações, submetidas a solicitações mecânicas de compressão uniaxial no próprio plano. Uma análise não-linear física e geométrica do comportamento pós-flambagem irá investigar a influência da espessura, comprimento da placa e tamanho do furo na resistência última.

\section{METODOLOGIA}

\subsection{Modelagem computacional}

A modelagem computacional foi realizada no software ANSYS,que é um programa baseado no método dos elementos finitos, e que pode ser utilizado nas mais diversas classes de problemas de engenharia.A modelagem da placa envolve a geração de um retângulo de tamanho $a \mathrm{x} b \mathrm{~mm}$. Para criara abertura é gerado um círculo com diâmetro $d_{c}$, cujas coordenadas $x$ e $y$ do centro coincidem com as da placa e são função de seu comprimento e largura 


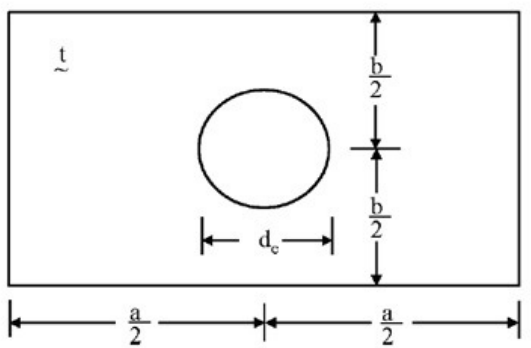

Figura 2.1 - Placa de aço com um orifício circular centrado submetida a compressão uniaxial. Adaptada (PAIK, 2007)

A divisão do domínio foi feita de acordo com o tamanho dos elementos atribuídos no comando "SizeControls", disponível em "Mesh Tool" no menu "Meshing".

Pode-se refinar a malha somente em algumas regiões, definidas por interesse de análise. No caso das placas com perfuração o refinamento foi feito no entorno do corte, onde há uma concentração de tensões (Figura 2.2).

Uma geometria inicial imperfeita é assumida conforme El-Sawy, Nazmy e

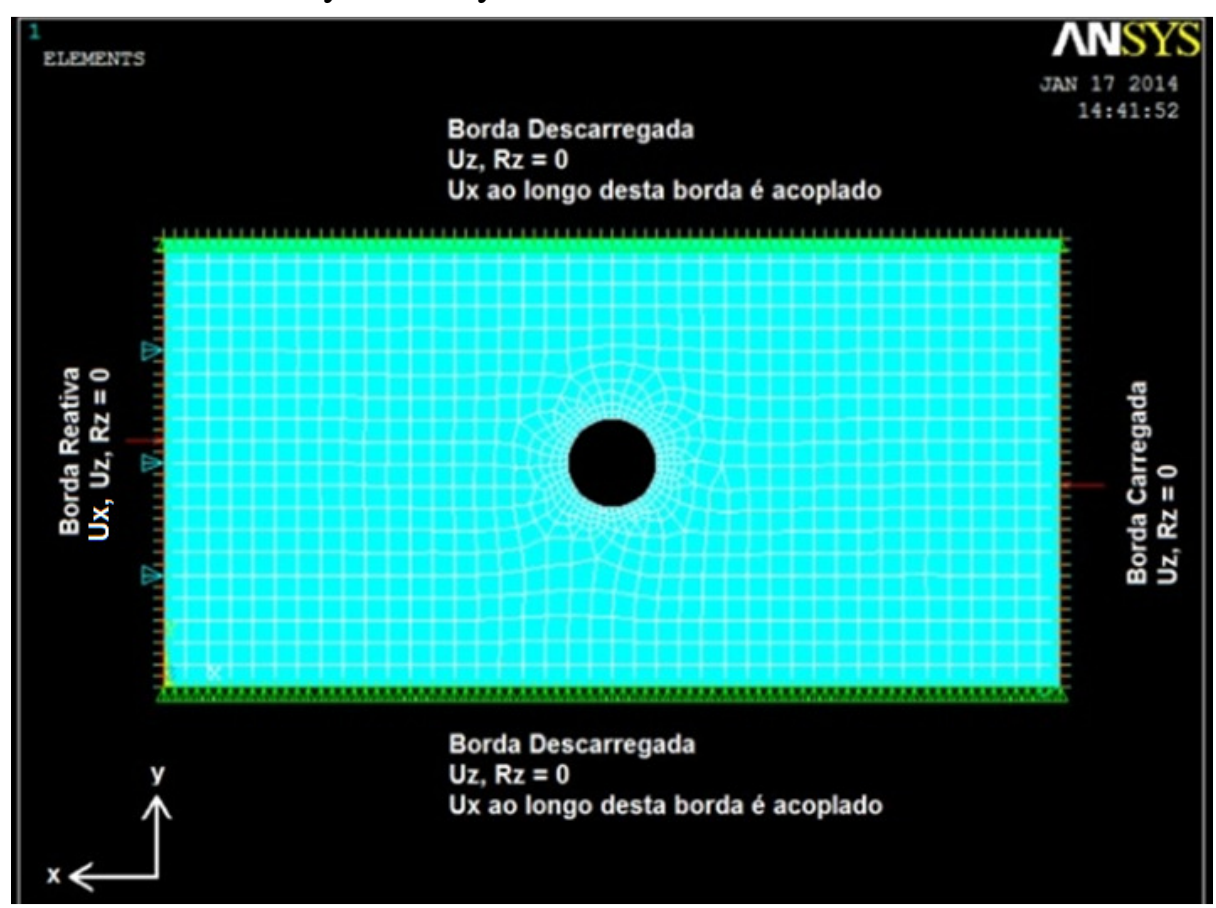

Figura 2.2 - Condições de contorno para modelagem das placas

A análise não linear com base no método dos elementos finitos utilizada é capaz de modelar a não-linearidade material, admitindo o aço como um material elasto-plástico perfeito, bem como a não-
Martini (2004), sendo este valor de imperfeição igual a $w_{0}=\frac{b}{2000}$, onde $b$ é a largura da placa. Conforme Kumar (2007), todas as bordas das placas foram consideradas como simplesmente apoiadas. Todos os nós ao longo das quatro bordas foram restringidos a deflexão e rotação ao longo da direção da espessura $(U Z, R Z=0)$.

As bordas descarregadas podem deformar no plano, mas permanecem em linha reta. Isto é obtido através do acoplamento de todos os nós ao longo das bordas descarregados no plano de deslocamento (UY), de tal modo que os deslocamentos ao longo do comprimento da placa são uniformes.Esta condição é para gerar a situação real da placa entre reforços longitudinais e transversais.A reação da borda é restringida para obter uma força igual causada devido à borda carregada. A extremidade reativa é restringida contra a deformação axial (UX = 0). 
equações de equilíbrio não-lineares são resolvidas utilizando processo de iteração Newton Raphson. O somatório da força axial em todos os nós ao longo da borda carregada para cada incremento de deslocamento fornece a carga última resistente da amostra.

\subsection{Teste de convergência}

A seguir serão apresentados dois testes de convergência que especificarão o tipo de elemento e o tamanho de malha que apresentaram um resultado mais satisfatório e um tempo hábil de processamento para serem aplicados neste trabalho.

Foram escolhidos dois tipos de elementos do software ANSYS a fim de identificar qual deles convergia ao resultado mais rápido: SHELL181 e SHELL93. Elementos do tipo Shell possuem grande aplicação na modelagem de estruturas finas, em que a espessura é pequena em comparação com as outras dimensões.

O elemento SHELL181 é um elemento que possui quatro nós, com seis graus de liberdade em cada nó (três rotações e três translações). Já o elemento SHELL93 possui oito nós, com seis graus de liberdade em cada nó (três rotações e três translações).

Foram feitos testes de convergência para os dois tipos de elementos para uma placa cujas características estão presentes na Tabela 2.1:

Tabela 2.1 - Características da placa modelo para teste de convergência do elemento

\begin{tabular}{|c|c|c|c|}
\hline $\mathrm{a}=\mathrm{b}(\mathrm{mm})$ & $\mathrm{t}(\mathrm{mm})$ & $\mathrm{d}(\mathrm{mm})$ & $\mathrm{d} / \mathrm{b}$ \\
\hline 125 & 1,615 & 25 & 0,2 \\
\hline $\mathrm{b} / \mathrm{t}$ & $\mathrm{w}_{0}(\mathrm{~mm})$ & $\mathrm{E}(\mathrm{GPa})$ & $\sigma \mathrm{y}(\mathrm{MPa})$ \\
\hline 77,4 & 0,097 & 210 & 323,3 \\
\hline
\end{tabular}

Os valores encontrados para os 4 testes realizados, onde o tamanho do elemento variou, serão apresentados a seguir Tabela 2.2.

Tabela 2.2 - Teste de convergência do tipo de elemento

\begin{tabular}{|c|c|c|c|c|c|c|c|}
\hline \multirow[b]{2}{*}{ Teste } & \multirow{2}{*}{$\begin{array}{l}\text { Tamanho } \\
\text { do } \\
\text { elemento } \\
(\mathrm{mm})\end{array}$} & \multicolumn{3}{|c|}{ SHELL93 } & \multicolumn{3}{|c|}{ SHELL181 } \\
\hline & & $\begin{array}{c}\text { PANSYS } \\
(\mathrm{kN})\end{array}$ & $\begin{array}{c}\text { Erro } \\
\text { relativo } \\
(\%)\end{array}$ & Aproximação & $\begin{array}{c}\text { PANSYS } \\
(\mathrm{kN})\end{array}$ & $\begin{array}{c}\text { Erro } \\
\text { relativo } \\
(\%)\end{array}$ & Aproximação \\
\hline 1 & $\mathrm{~b} / 5$ & 37,8709 & 0,0011 & 0,9989 & 38,4436 & 0,0096 & 0,9904 \\
\hline 2 & $b / 10$ & 37,819 & 0,0025 & 0,9975 & 38,4383 & 0,0095 & 0,9905 \\
\hline 3 & $\mathrm{~b} / 20$ & 37,9039 & 0,0002 & 0,9998 & 38,1549 & 0,0021 & 0,9979 \\
\hline 4 & $\mathrm{~b} / 40$ & 37,9122 & 0,0000 & 1,0000 & 38,0762 & 0,0000 & 1,0000 \\
\hline
\end{tabular}




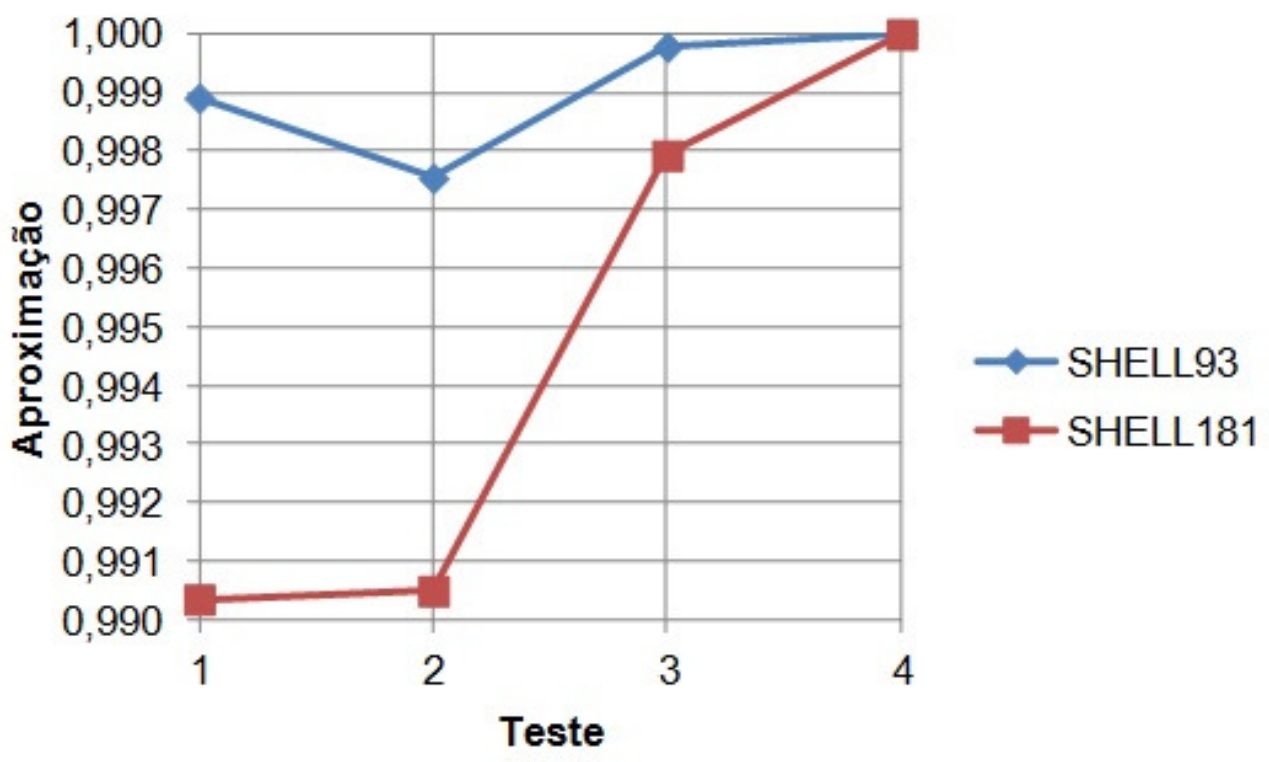

Figura 2.3 - Teste de convergência do tipo de elemento

O tempo de processamento para o elemento SHELL93 foi maior que o SHELL181, mas com uma malha menor, o SHELL93 produz resultados mais satisfatórios que o SHELL181. Desta forma o tipo de elemento escolhido pararealizar a modelagem computacional foio SHELL93.

Foram feitos também testes de convergência de malha para dois tipos de placas (A e B) cujas características estão presentes na Erro! Fonte de referência não encontrada..
Tabela 2.3 - Características das placas modelo para teste de convergência de mala

\begin{tabular}{|c|c|c|c|c|c|c|}
\hline Placa & $\begin{array}{c}\mathrm{a}=\mathrm{b} \\
(\mathrm{mm})\end{array}$ & $\begin{array}{c}\mathrm{t} \\
(\mathrm{mm})\end{array}$ & $\begin{array}{c}\mathrm{d} \\
(\mathrm{mm})\end{array}$ & $\begin{array}{c}\mathrm{w}_{0} \\
(\mathrm{~mm})\end{array}$ & $\begin{array}{c}\mathrm{E} \\
(\mathrm{GPa})\end{array}$ & $\begin{array}{c}\sigma \mathrm{M} \\
(\mathrm{MPa})\end{array}$ \\
\hline A & 125 & 1,615 & 0 & 0,229 & 210 & 323,3 \\
\hline B & 125 & 1,615 & 25 & 0,097 & 210 & 323,3 \\
\hline
\end{tabular}

Os testes e os respectivos resultados serão apresentados a seguir.

Tabela 2.4 - Teste de convergência de malha para a placa A

\begin{tabular}{|c|c|c|c|c|c|}
\hline Teste & $\begin{array}{c}\mathrm{a}=\mathrm{b} \\
{[\mathrm{mm}]}\end{array}$ & $\begin{array}{c}\text { Tamanho } \\
\text { do } \\
\text { elemento } \\
(\mathrm{mm})\end{array}$ & $\begin{array}{c}\text { Pul } \\
\text { ANSYS } \\
{[\mathrm{kN}]}\end{array}$ & $\begin{array}{c}\text { Erro } \\
\text { Relativo } \\
(\%)\end{array}$ & Aproximação \\
\hline 1 & 125 & $\mathrm{~b} / 5$ & 40,4388 & 0,377 & 0,623 \\
\hline 2 & 125 & $\mathrm{~b} / 10$ & 40,5781 & 0,034 & 0,966 \\
\hline 3 & 125 & $\mathrm{~b} / 20$ & 40,578 & 0,034 & 0,966 \\
\hline 4 & 125 & $\mathrm{~b} / 40$ & 40,5919 & 0,000 & 1,000 \\
\hline
\end{tabular}




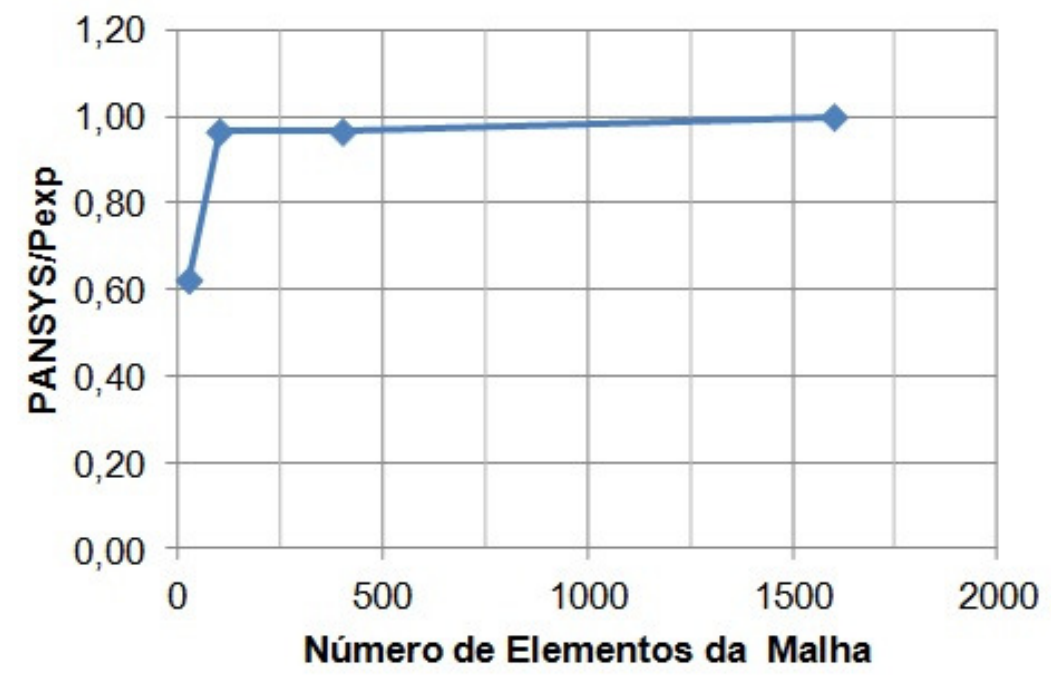

Figura 2.4 - Teste de convergência de malha para a placa A

Tabela 2.5 - Teste de convergência de malha para a placa B

\begin{tabular}{|c|c|c|c|c|c|}
\hline Teste & $\begin{array}{c}\mathrm{a}=\mathrm{b} \\
{[\mathrm{mm}]}\end{array}$ & $\begin{array}{c}\text { Tamanho } \\
\text { do } \\
\text { elemento } \\
(\mathrm{mm})\end{array}$ & $\begin{array}{c}\text { Pul } \\
\text { ANSYS } \\
{[\mathrm{kN}]}\end{array}$ & $\begin{array}{c}\text { Erro } \\
\text { Relativo } \\
(\%)\end{array}$ & Aproximação \\
\hline 1 & 125 & $\mathrm{~b} / 5$ & 37,8709 & 0,109 & 0,891 \\
\hline 2 & 125 & $\mathrm{~b} / 10$ & 37,8190 & 0,246 & 0,754 \\
\hline 3 & 125 & $\mathrm{~b} / 20$ & 37,9039 & 0,022 & 0,978 \\
\hline 4 & 125 & $\mathrm{~b} / 40$ & 37,9122 & 0,000 & 1,000 \\
\hline
\end{tabular}

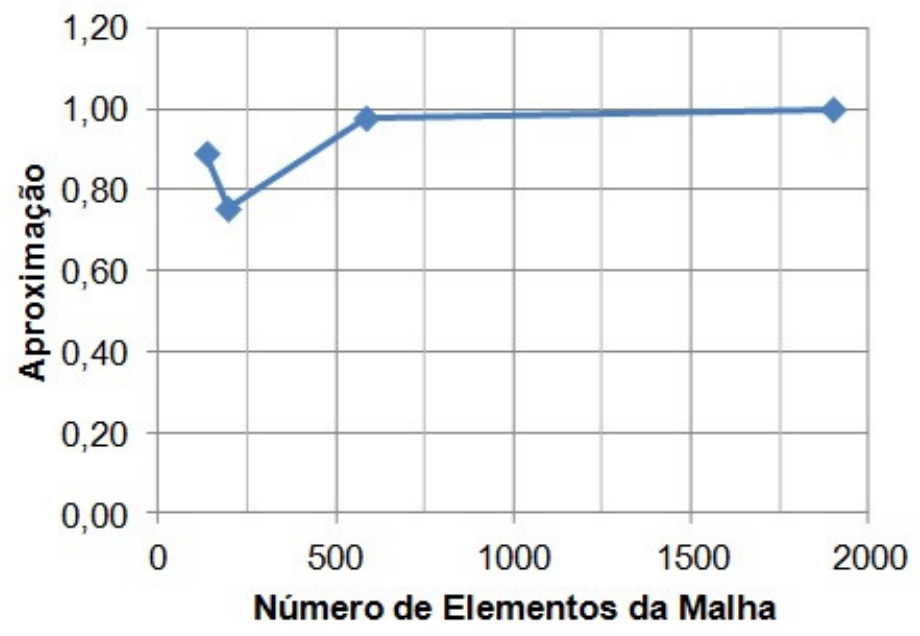

Figura 2.5 - Teste de convergência de malha para a placa B

É importante saber a condição de refinamento do modelo numérico em virtude dos resultados que se pretende obter e do tempo disponível para realizar o estudo. Os valores obtidos no Teste 4 foram tomados como referência para determinação do erro relativo.Observando os gráficos, nota-se que o mais indicado seria realizar análises como as do Teste 4, com a malha mais refinada e com elementos do tamanho correspondente a largura da placa dividido por 40 .

Tendo em vista que o tempo de processamento do software para 
apresentar os valores do Teste 4 não foram satisfatórios para a execução do trabalho, os testes serão simulados conforme o Teste 3 , onde a malha possui elementos com tamanho b/20.

\subsection{Validação do modelo}

A validação do modelo é realizada comparando-se a carga máxima encontrada através do modelo numérico rodado no software ANSYS com a carga máxima mostrada na Erro! Fonte de referência não encontrada., encontrada em testes experimentais realizados por NARAYANAN E CHOW (1984).

Tabela 2.6 - Valores experimentais (NARAYANAN e CHOW, 1984)

\begin{tabular}{|c|c|c|c|c|c|c|c|c|c|}
\hline & $\begin{array}{c}\mathrm{a}=\mathrm{b} \\
(\mathrm{mm})\end{array}$ & $\mathrm{t}(\mathrm{mm})$ & $\begin{array}{c}\mathrm{d} \\
(\mathrm{mm})\end{array}$ & $\mathrm{d} / \mathrm{b}$ & $\mathrm{b} / \mathrm{t}$ & $\begin{array}{c}\mathrm{w}_{0} \\
(\mathrm{~mm})\end{array}$ & $\begin{array}{c}\mathrm{E} \\
(\mathrm{GPa})\end{array}$ & $\sigma \mathrm{y}(\mathrm{MPa})$ & $\mathrm{P}_{\text {ult }}(\mathrm{kN})$ \\
\hline PL1a & 125 & 1,615 & 0 & 0,0 & 77,40 & 0,229 & 210 & 323,3 & 39,32 \\
CIR2a & 125 & 1,615 & 25 & 0,2 & 77,40 & 0,229 & 210 & 323,3 & 37,46 \\
CIR2b & 125 & 1,615 & 25 & 0,2 & 77,40 & 0,097 & 210 & 323,3 & 38,7 \\
CIR3a & 125 & 1,615 & 37,5 & 0,3 & 77,40 & 0,136 & 210 & 323,3 & 33,94 \\
CIR4a & 125 & 1,615 & 50 & 0,4 & 77,40 & 0,304 & 210 & 323,3 & 29,57 \\
CIR4b & 125 & 1,615 & 50 & 0,4 & 77,40 & 0,127 & 210 & 323,3 & 28,39 \\
Cir5a & 125 & 1,615 & 62,5 & 0,5 & 77,40 & 0,279 & 210 & 323,3 & 27,35 \\
Cir6 & 86 & 2,032 & 25 & 0,3 & 42,32 & 0,254 & 210 & 334,7 & 42,17 \\
Cir7 & 86 & 1,615 & 25 & 0,3 & 53,25 & 0,229 & 210 & 323,3 & 26,18 \\
Cir8 & 86 & 0,972 & 25 & 0,3 & 88,48 & 0,102 & 210 & 317,6 & 12,35 \\
Cir9 & 86 & 0,693 & 25 & 0,3 & 124,10 & 0,051 & 210 & 322,8 & 7,33 \\
Cir10 & 86 & 2,032 & 40 & 0,5 & 42,32 & 0,102 & 210 & 334,7 & 33,64 \\
Cir11 & 86 & 1,615 & 40 & 0,5 & 53,25 & 0,279 & 210 & 323,3 & 22,14 \\
Cir12 & 86 & 0,972 & 40 & 0,5 & 88,48 & 0,152 & 210 & 317,6 & 10,89 \\
\hline
\end{tabular}

O elemento utilizado na modelagem foi o Shell 93 e a malha de elementos finitos possui tamanho igual a $b / 20$, onde $b$ é a largura da placa.

A comparação dos resultados experimentais obtido por NARAYANAN
E CHOW (1984) para a capacidade de carga máxima de placas perfuradas com os valores correspondentes obtidos a partir da análise através do software ANSYS, dada na Tabela 2.7, mostra que existe uma boa concordância entre os dois conjuntos de resultados. 
Tabela 2.7 - Comparação dos resultados

\begin{tabular}{|c|c|c|c|}
\hline PLACA & $\mathrm{P}_{\text {ult,Exp }}(\mathrm{kN})$ & $\begin{array}{c}\mathrm{P}_{\text {ult,ANSYS }} \\
(\mathrm{kN})\end{array}$ & $\mathrm{P}_{\text {ANSYS }} / \mathrm{P}_{\text {Exp }}$ \\
\hline PL1a & 39,32 & 40,58 & 1,03 \\
CIR2a & 37,46 & 37,63 & 1,00 \\
CIR2b & 38,7 & 37,90 & 0,98 \\
CIR3a & 33,94 & 35,03 & 1,03 \\
CIR4a & 29,57 & 31,52 & 1,07 \\
CIR4b & 28,39 & 31,72 & 1,12 \\
Cir5a & 27,35 & 28,07 & 1,03 \\
Cir6 & 42,17 & 40,15 & 0,95 \\
Cir7 & 26,18 & 28,10 & 1,07 \\
Cir8 & 12,35 & 13,63 & 1,10 \\
Cir9 & 7,33 & 8,86 & 1,21 \\
Cir10 & 33,64 & 34,24 & 1,02 \\
Cir11 & 22,14 & 23,21 & 1,05 \\
Cir12 & 10,89 & 11,38 & 1,04 \\
\hline
\end{tabular}

A subestimação ou superestimação pelo modelo numérico está dentro de um nível aceitável, onde o valor da relação $\mathrm{P}_{\text {AnSYS }} / \mathrm{P}_{\text {Exp }}$ médio é 1,05 , com um desvio padrão de 0,0641 e um coeficiente de variação de $6,10 \%$.
Para a placa CIR2b é apresentado o gráfico comparativo entre os valores experimentais e o da simulação numérica no programa ANSYS ao longo da analise, onde podemos observar que a capacidade de carga é praticamente coincidente no pico da curva. (Figura 2.6)

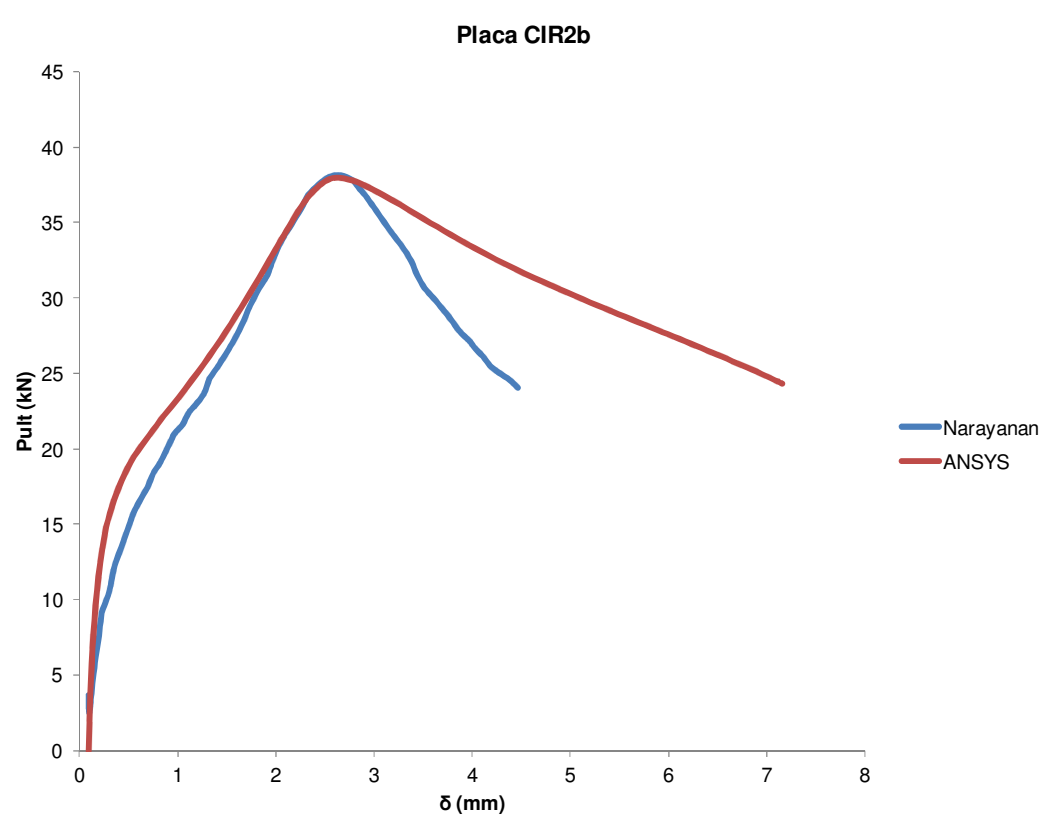

Figura 2.6 - Comparação dos resultados para a placa CIR2b

Pode-se concluir que o modelo numérico baseado no método dos elementos finitos utilizado é capaz de modelar o comportamento e prever a capacidade de carga de placas perfuradas com precisão suficiente. 


\subsection{Estudo paramétrico}

As situações analisadas neste trabalho compreendem placas quadradas e retangulares, com ou sem perfuração, submetidas à compressão uniaxial, onde $a$ é o comprimento, bé a largura, té a espessura, $d$ é o diâmetro do furo, $E$ é o módulo de elasticidade do aço de 210 GPa, $v$ é o coeficiente de Poisson de 0,3 . O material utilizado foi o aço AH36 com tensão de escoamento $\sigma \mathrm{y}=355 \mathrm{MPa}$.

Os testes foram divididos em seis grupos, onde cada um apresenta variações paramétricas no tamanho da placa, na espessura e no tamanho do furo. Os valores característicos estão apresentados na Tabela 2.8 até Tabela 2.10 .

Tabela 2.8 - Variações paramétricas de comprimento

\begin{tabular}{|c|c|c|c|}
\hline & $\mathrm{a}(\mathrm{mm})$ & $\mathrm{b}(\mathrm{mm})$ & $\mathrm{a} / \mathrm{b}$ \\
\hline GRUPO 1 & 900 & 900 & 1 \\
\hline GRUPO 2 & 1800 & 900 & 2 \\
\hline GRUPO 3 & 2700 & 900 & 3 \\
\hline GRUPO 4 & 3600 & 900 & 4 \\
\hline GRUPO 5 & 4500 & 900 & 5 \\
\hline GRUPO 6 & 5400 & 900 & 6 \\
\hline
\end{tabular}

O coeficiente $\beta$ é definido como índice de esbeltez da placa e dado por (Paik,2007):

$$
\beta=\frac{b}{t} \sqrt{\frac{E}{\sigma y}}
$$

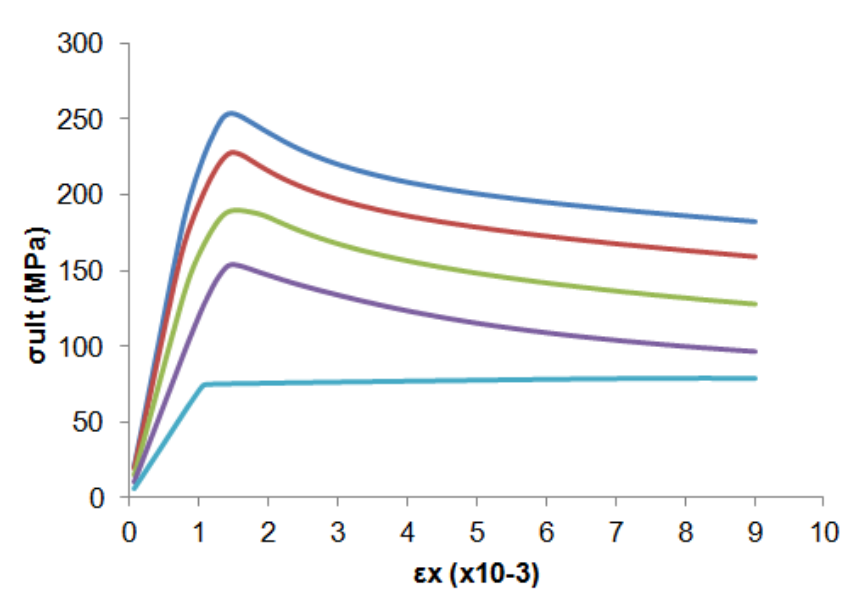

\section{RESULTADOS}

\subsection{Influência do furo}

A seguir serão apresentados gráficos da tensão última, obtida pela divisão da força última do ANSYS pela área $b \times t$ da seção transversal da placa, versus a deformação na direção $\mathrm{x}\left(\varepsilon_{x}\right)$, consequente dos incrementos de deslocamento $\mathrm{U}_{\mathrm{X}}$ ao longo da borda carregada (em $\mathrm{x}=0$ ), para uma chapas de aço cuja relação $a / b=1$, com um único furo circular centrado até e após a força máxima ser atingida, quando o tamanho do orifício (diâmetro) é variado.

Figura 3.1 - Influência do furo em placa com relação $a / b=1$ e $t=15 \mathrm{~mm}$ 
É evidente, a partir da analise da Figura 3.1 Erro! Fonte de referência não encontrada.que a força máxima é significativamente reduzida pelo furo. Quando a área do orifício circular corresponde a $50,3 \%$ da área total da $\operatorname{placa}(d c / b=0,8$ e $\beta=1,46)$, a força máxima da placa é reduzida em aproximadamente $\quad 68,9 \% \quad$ quando comparada com a placa maciça.

\subsection{Efeito da esbeltez}

A esbeltez de uma placa é um fator de forte influência na forma de como a estrutura irá sofrer flambagem, definindo se esta será elástica ou inelástica, além de determinar a carga de ruptura. Quanto mais robusta for a placa, mais resistente à flambagem ela será, o que é comprovado quando analisamos o gráfico da Figura 3.2 .

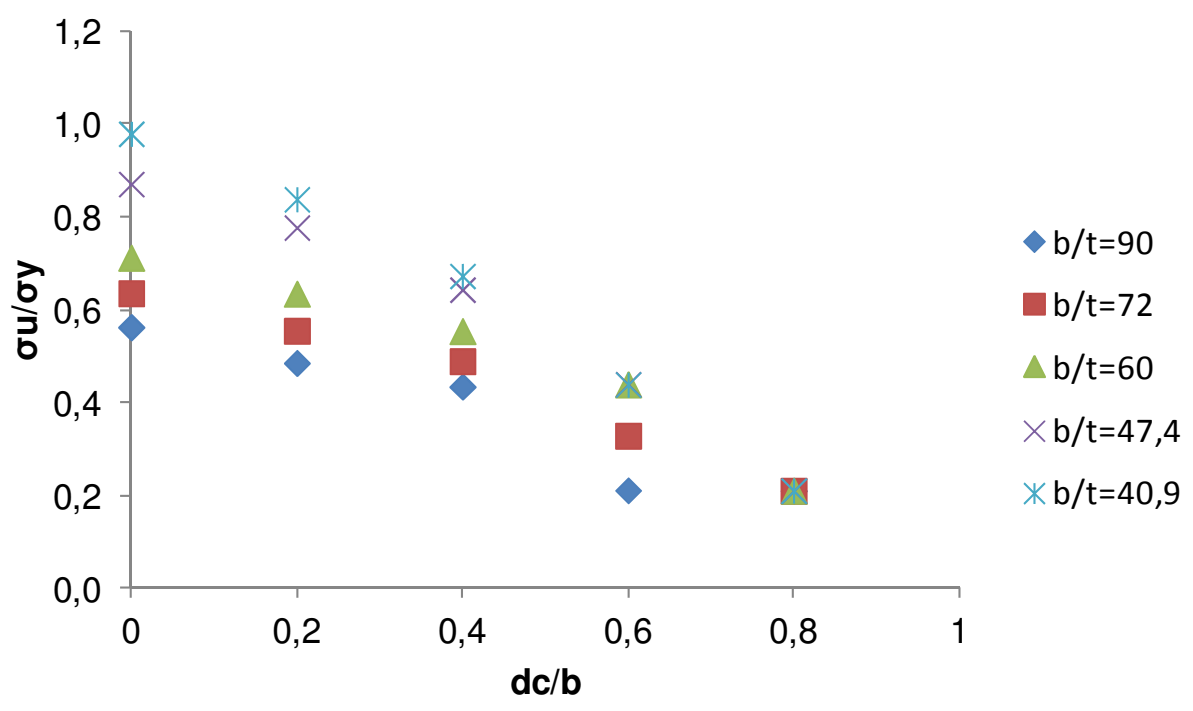

Figura 3.2 - Efeito da esbeltez para placa $a / b=4$

Para as placas ditas maciças, ou seja, sem furos, a esbeltez da placa é determinante no valor de sua resistência última. Notase que quanto menor a relação $b / t$, mais espessa a placa, e maior sua resistência. À medida que o diâmetro do furo vai aumentando, há uma convergência para o valor da carga última, independentemente da espessura da placa. Tal convergência deve-se ao motivo de a carga última ser limitada pela tensão de escoamento do material e pela redução da seção transversal da placa causada pela presença do furo.

\subsection{Variações no parâmetro de proporção $a / b$}

Foram feitos testes com variação no parâmetro de proporção $a / b$ das placas. O gráfico da Figura 3.3 mostra os resultados obtidos para uma placa com espessura de $22 \mathrm{~mm}$. É interessante notar que a resistência última da placa é raramente afetada pelos aspectos de proporção. 


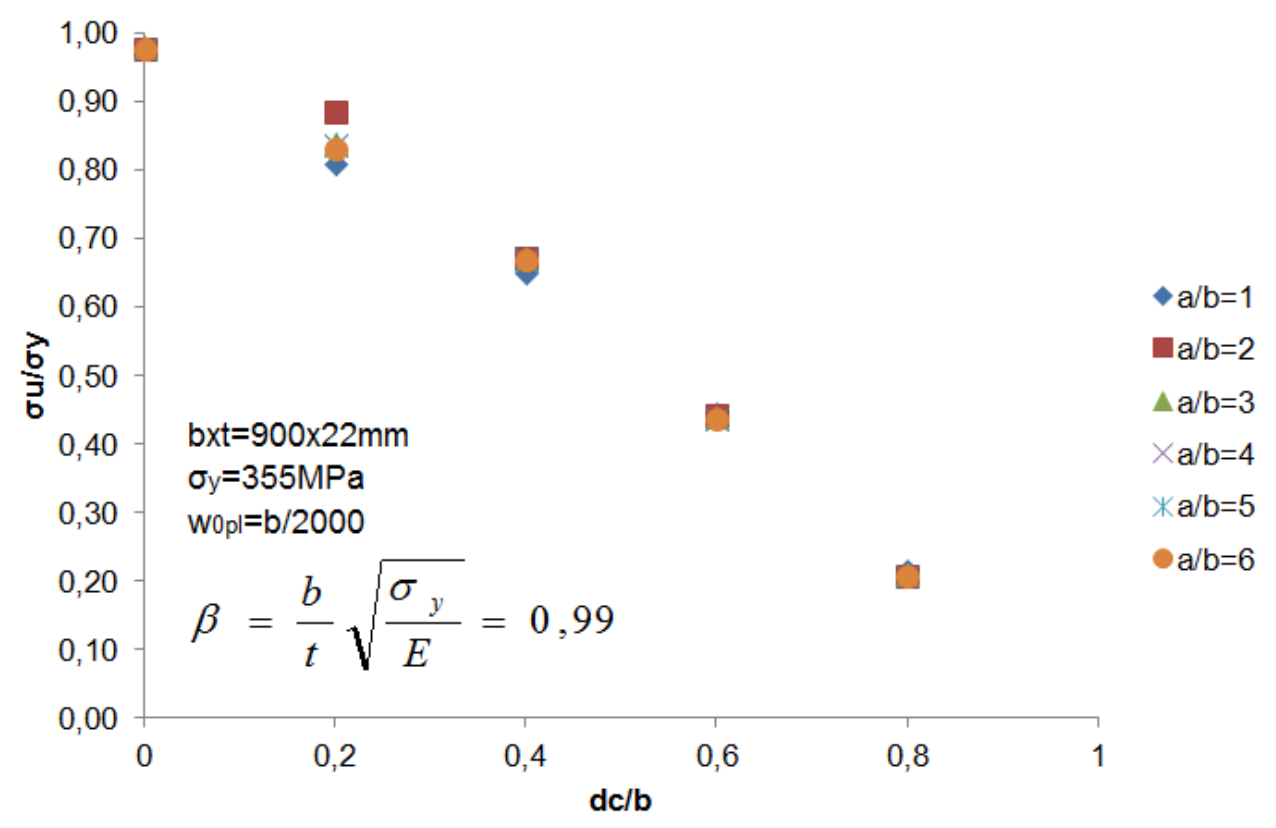

Figura 3.3 - Gráfico com variação no parâmetro de proporção para placa com t=22mm

\section{CONCLUSÕES}

Ficou evidente que as placas maciças, ou seja, sem furo, possuem uma resistência última superior às placas perfuradas, e que a força máxima é significativamente reduzida pelo furo. Nota-se também que quanto mais espessa a placa, maior sua resistência. À medida que o diâmetro do furo vai aumentando, há uma convergência para o valor da tensão última, independentemente da espessura da placa. $\mathrm{Na}$ análise das variações paramétricas de proporção conclui-se que a resistência última da placa é raramente afetada com a variação de seu comprimento.

\section{REFERÊNCIAS}

BLEICH, F. Theorie und berechnung der eisernen brücken. [S.1.]: Julius Springer, 1924.

BRYAN, G. H. On the stability of a plane under thrusts in its own plane with applications to the buckling of the sides of a ship. [S.1.]: Proceedings of the London Mathematical Society, v. 22, 1891.

EL-SAWY, K. M.; NAZMY, A. S.; MARTINI, M. I. Elasto-plastic buckling of perforated plates under uniaxial compression. Thin-Walled Structures, v. 42, p. 1083-1101, 2004.

KUMAR, M. S. ULTIMATE STRENGTH OF SQUARE PLATE WITH RECTANGULAR OPENING UNDER AXIAL COMPRESSION. Journal of Naval Architecture and Marine Engineering, Junho 2007.

NARAYANAN, R.; CHOW, F. Y. Ultimate Capacity of Uniaxially Compressed Perforated. [S.1.]. 1984.

PAIK, J. K.; THAYAMBALLI, A. K.; KIM, B. J. Advanced Ultimate Strength Formulations for Ship Plating under Combined Biaxial Compression/Tension, Edge Shear, and Lateral Pressure Loads. Marine Technology, v. 38, p. 9-25, 2001.

ROBERTS, T. M.; AZIZIAN, Z. G. Strength of Perforated Plates Subjected to In-Plane Loading. Thin-Walled Structures, v. II, p. 153-164, 1984.

ROORDA, J. Buckling of elastic structures. Canadá: Solid Mechanics Division, University of Waterloo Press, 1980. 
SAINT-VENANT, A. J. C. B. D. Théorie de l'elasticité des corps solides. Paris: Clebsch, 1883.

SCHUMAN, L.; BACK, G. Strength of retangular plates under edge compression. EUA: NASA Technical Report, TR, R-40, 1959.

SHANMUGAM, N. E. Openings in Thin-Walled Steel Structures. ThinWalled Structures, v. 28, p. 355-372, 1997.

SHANMUGAM, $\quad \mathrm{N}$. $\quad$ E.; THEVENDRAN, V.; TAN, Y. H. Design Formula for Axially Compressed Perforated Plates. Thin-Walled Structures, v. 34, p. 1-20, 1999.

TIMOSHENKO, S. P. Einige stabilitätsprobleme der elastizitätstheorie. [S.1.]: Zeitschrift für Mathematik und Physik, v. 58, 1910.

TRAHAIR, N. S.; BRADFORD, M. A. The behavior and design of steel structures. $2^{\mathrm{a}}$ Edição. ed. [S.l.]: Chapman \& Hall, 1988.

YETTRAM, A. L.; BROWN, C. J. The Elastic Stability of Square Perforated Plates. Computers and Structures, v. 21, p. 1267-1272, 1985. 